\title{
Timing of intervention in acute pancreatitis
}

\author{
C.D. Johnson
}

University Surgical Unit, F Level, Centre Block, Southampton General Hospital, Tremona Road, Southampton SO9 $4 X Y$, UK

Summary: This review examines the appropriate timing of intervention in acute pancreatitis. In gallstone pancreatitis, it is now clear that cholecystectomy during the primary admission carries no greater risk of complications than delayed cholecystectomy and enables earlier recovery to normal activity. This course of action pre-empts a second, possibly fatal attack of acute pancreatitis. Cholecystectomy should be done after the acute phase has settled, before discharge from hospital. Patients with gallstones should now be offered endoscopic sphincterotomy within $\mathbf{4 8}$ hours of admission. This approach is safe, and reduces the risk of complications.

When complications develop, early necrosectomy is only indicated if conservative measures fail. Delayed ( $>10$ days) necrosectomy is appropriate if there is evidence of sepsis, or clinical failure to improve. Pancreatic pseudocysts can often be managed expectantly; a high proportion will resolve spontaneously. After a delay of 12 weeks, persistent cysts require evaluation by endoscopic pancreatography, which gives crucial information in the choice between percutaneous or surgical drainage of the pseudocyst.

A patient with pancreatitis is usually treated under the care of a surgeon, who has traditionally taken the decision on the timing of any intervention, and has performed such intervention at open operation. Recently, the development of alternative techniques has enabled the surgeon to call on the skills of his colleagues in endoscopy and interventional radiology. However, the availability of these alternatives to surgery should not affect the timing of intervention unless it can be clearly shown that such a change in timing combined with the minimally invasive technique can improve the outcome for the patient.

Intervention may be required to deal with gallstones in the gallbladder or in the bile duct, to deal with, or ideally prevent, the deleterious systemic effects of pancreatic and peripancreatic necrosis, or to drain a peripancreatic abscess. Peripancreatic fluid collections and pancreatic pseudocysts may also require either internal or external drainage to relieve symptoms or prevent complications.

\section{Gallbladder gallstones}

A patient with pancreatitis should undergo ultrasonography of the gallbladder within 24 hours of admission to hospital, primarily to facilitate the early management of stones in the common bile duct (see below). This early diagnosis of gallstones also enables the surgeon to formulate a plan of management for the stones in the gallbladder. Although in practice this must be linked to management of stones in the bile duct, the arguments will be discussed separately.

It is generally accepted that if a patient has gallstones and has developed an attack of acute pancreatitis, then cholecystectomy will be required to prevent the development of further attacks. The traditional approach has been to allow the patient

Correspondence: C.D. Johnson, M.Chir., F.R.C.S.

Received: 9 December 1992 to recover from the attack of pancreatitis, to discharge the patient from hospital, and to perform cholecystectomy 6-12 weeks later. In 1974, Acosta and Ledesma ${ }^{1}$ demonstrated that nearly all patients with gallstones and acute pancreatitis had evidence of migration of stones through the bile duct. They and others subsequently challenged the traditional delayed approach to cholecystectomy and recommended surgery during the primary admission with acute pancreatitis. ${ }^{2-4}$ The overall risk of complications or death is equivalent or better with early rather than delayed surgery, and it is now accepted that cholecystectomy should be performed during this first admission. ${ }^{5,6}$

The anxiety that early operation might be more difficult as a result of peripancreatic inflammation appears to be unfounded. Biliary surgery was uncomplicated in 19 patients who presented with an elevated amylase and upper abdominal pain and who underwent laparotomy within 7 days of emergency admission. ${ }^{7}$ This observation is confirmed by the experience of surgeons who perform 
cholecystectomy during the primary admission, after resolution of acute signs. There can be no justification for sending a patient who has had acute pancreatitis away from hospital with gallstones still in place. Such a course exposes the patient to the unnecessary risk of a further potentially fatal attack of acute pancreatitis.

The relative simplicity of cholecystectomy after uncomplicated acute pancreatitis suggests that laparoscopic cholecystectomy should be attempted in these patients. One might suppose that the risk of conversion to open surgery is higher in this group, but a preliminary report suggests that this is not the case. ${ }^{8}$ However, it is essential to obtain cholangiography before or during the procedure in order to identify and deal with common bile duct stones.

\section{Immediate or early cholecystectomy}

While there can be no doubt that cholecystectomy within the first admission, after resolution of symptoms, is advantageous for the patient, there remains controversy over the timing of cholecystectomy within the first admission. The reason for this controversy lies in the possibility of removing stones from the common bile duct at the same time as performing cholecystectomy. The separation of these two steps following the introduction of endoscopic sphincterotomy has certainly reduced the potential advantages of immediate cholecystectomy (within 48 hours of admission).

Most common duct stones pass spontaneously and do not require operation and patients with mild pancreatitis which will resolve spontaneously do not require urgent intervention. In contrast, operation in an unstable patient shortly after admission to hospital may lead to unnecessary complications. Unfortunately few randomized studies address this question and a judgement must be made on the basis of uncontrolled, often small series. The reported morbidity and mortality vary widely with no difference between the two approaches. One study reported a mortality in excess of $30 \%$ both in patients operated within 72 hours of onset of symptoms and those in whom operation was delayed. Others report mortality approaching zero with both techniques, and the median figure for mortality lies in the range of $6-8 \% .^{5}$

In one non-randomized study ${ }^{9}$ operation was performed within 48 hours of admission, after 3-7 days or after more than 7 days. The patients in each group had similar severity of pancreatitis and there was no difference in mortality or complication rates. However, these were both low which make it difficult to detect differences between the groups. One randomized study ${ }^{10}$ has compared cholecystectomy within 48 hours with the result of surgery performed between the fourth and tenth day after admission. Immediate surgery had much higher morbidity and mortality $(30 \%$ and $15 \%)$ than delayed surgery (5\% and $2 \%)$.

The advice to be given on the management of gallbladder gallstones is clear. The safest course is to allow the pancreatitis to settle and then to perform cholectystectomy before the patient is discharged from hospital. This approach may be varied if the patient is unwell and requires surgery for complications of the pancreatitis. In this case, cholecystectomy should be performed at the time of pancreatic debridement. It may be safe to delay cholecystectomy if endoscopic sphincterotomy and bile duct clearance have been performed during the primary admission, but there are no data to confirm this. Where laparoscopic cholecystectomy is available it will probably be the procedure of choice for cholecystectomy after pancreatitis but further results are required to support this approach. All patients who present with pancreatitis and stones in the gallbladder should undergo cholangiography either before or during cholecystectomy to detect and allow extraction of stones in the common bile duct.

\section{Bile duct stones}

Gallstones cause pancreatitis as they migrate down the cystic duct and through the sphincter of Oddi The exact mechanism of this is unknown, but it is reasonable to suppose that the persistence of the stone or stones in the bile duct, or impaction at the ampulla may add fuel to the fire that is already burning in the pancreas. Several authors have therefore proposed early surgery, within 24 or 48 hours of admission to hospital, to clear the bile duct and prevent serious complications of the pancreatitis. However, as noted above, this approach in the hands of many surgeons leads to increased morbidity and mortality. Furthermore, the incidence of positive duct exploration is far from $100 \%$ in all of these series. Thus some patients are exposed to the risks of early surgery, when they are still unwell from the pancreatitis, with no conceivable benefit, as the stone has already left the bile duct. Thus despite its theoretical advantages, surgical bile duct exploration immediately after admission has proved disappointing.

The advent of endoscopic retrograde cholangiography (ERC) and associated endoscopic sphincterotomy (ES) has allowed a more selective and less dangerous approach to common bile duct stones.

The use of these techniques at an early stage in acute pancreatitis was first proposed in 1981. ${ }^{11}$ These and subsequent authors have emphasized the need to avoid excessive manipulation of the papilla, 
and if possible to avoid injection into the pancreatic duct. There have now been three controlled studies which all demonstrate the benefit of early ES in gallstone pancreatitis (Table I). The clear message from these studies is that early ERC with ES if there are stones in the duct is safe, and reduces morbidity in biliary pancreatitis. All patients suspected of biliary pancreatitis should undergo ERC within 24 to 48 hours of admission. The need may be less pressing in those with mild pancreatitis ${ }^{12}$ but this cannot be deduced from the data presented in two of the studies. All studies show a clear reduction in morbidity and mortality in patients with confirmed gallstones. Adding together the three series there were 203 such patients who had early ERC and ES. Twenty-eight developed complications and three died. In contrast 173 patients had delayed ERC and ES. Forty-four complications were noted and 14 patients died. Fan et al. ${ }^{14}$ found no biliary sepsis in patients who underwent early ERC and ES, compared with four of 58 with predicted mild pancreatitis and eight of 40 with predicted severe pancreatitis. There was no excess morbidity associated with ERC in those with mild pancreatitis in any of the three studies.

There are alternatives to endoscopic sphincterotomy which may allow extraction of bile duct stones without permanent destruction of the sphincter of Oddi. These include pharmacological sphincter relaxation using nitrates or anticholinergic agents, or alternatively balloon dilatation of the ampulla. ${ }^{15}$ Further evaluation of these techniques is awaited.

\section{Pancreatic necrosis}

Pancreatic and peripancreatic necrosis can be diagnosed by bolus intravenous contrast-enhanced computed tomography (CT) at an early stage in the disease, ${ }^{16}$ but the sensitivity of this investigation improves up to one week after surgery. ${ }^{17}$ The operative management of pancreatic necrosis is generally easier when 7-10 days have elapsed since onset of symptoms, after demarcation has taken place in the peripancreatic tissues. Our policy is to perform contrast-enhanced CT one week after admission if there are signs or symptoms of continuing pancreatic inflammation (fever, leucocytosis, abdominal pain, vomiting). If the patient fails to respond to full intensive care measures and resuscitation during the early days of the attack, then CT is performed immediately as a guide to surgical exploration.

Pancreatic necrosis may become infected. Overall, $40 \%$ of patients have evidence of infection, and the incidence is highest in the third week from onset of symptoms. ${ }^{18}$ Percutaneous CT-guided aspiration is a reliable technique to demonstrate infection..$^{19,20}$ and it has been suggested that this technique can be used to predict the need for surgical intervention. ${ }^{21}$ Bradley and Allen ${ }^{22}$ reported 38 patients with pancreatic and peripancreatic necrosis. Twenty-seven of these patients were found to have infection in the necrotic tissue and all required operation. Only one of the remaining 11 patients without infection required operation, all survived. This confirms a well-recognized clinical observation that patients with extensive peripancreatic necrosis and failure of pancreatic enhancement on CT may recover from the acute attack, become asymptomatic, and may never require surgical intervention. There are thus two main indications for operation: clinical criteria, and bacteriological confirmation of infected necrosis.

There are numerous reports of the management of pancreatic and peripancreatic necrosis. The timing of surgery in these patients reflects the authors' enthusiasm for early aggressive interven-

Table I Outcome of early or delayed ERC + ES in patients with suspected biliary pancreatitis. Results of three randomized studies

\begin{tabular}{|c|c|c|c|c|c|c|c|c|c|}
\hline & \multicolumn{3}{|c|}{ Mild } & \multicolumn{3}{|c|}{ Severe } & \multicolumn{3}{|c|}{ All cases } \\
\hline & $\mathrm{n}$ & Comp. & Death & $\mathrm{n}$ & Comp. & Death & $\mathbf{n}$ & Comp. & Death \\
\hline \multicolumn{10}{|l|}{ Neoptolemos et al. ${ }^{12}$} \\
\hline$<48$ hours & 28 & 4 & 0 & 22 & 4 & 0 & 59 & 10 & 1 \\
\hline$>5$ days & 29 & 4 & 0 & 24 & 13 & 3 & 62 & 21 & 5 \\
\hline \multicolumn{10}{|l|}{ Nowak et al. . $^{13}$} \\
\hline$<24$ hours & & & & & & & 56 & 8 & 0 \\
\hline None & & & & & & & 57 & 16 & 6 \\
\hline \multicolumn{10}{|l|}{ Fan et al..$^{14}$} \\
\hline$<24$ hours & 56 & 10 & 0 & 41 & $26^{*}$ & 5 & 97 & $36^{*}$ & 5 \\
\hline Delayed selective & 58 & 7 & 0 & 40 & $49^{*}$ & 9 & 98 & $56^{*}$ & 9 \\
\hline
\end{tabular}

*More than one per patient. 
tion or preference for delay. It is difficult to compare many of these series as it is often not clear what is the population from which the patients are selected for operation. However, three studies contain sufficient information to illustrate the results of contrasting approaches.

A report from Glasgow ${ }^{23}$ reflected the usual British practice. The authors operated on 21 patients for pancreatic necrosis from a total consecutive series of 456 patients with acute pancreatitis. Surgery was delayed at least until the second week of admission whenever possible. The operative mortality was high (eight patients, $38 \%$ ). Operation was only deemed necessary when the surgeon felt that an adequate trial of conservative management had failed. Surgery was performed a median of 21 days after admission to hospital (range 1-150). The indication for surgery was a persistent pyrexia or leucocytosis (10 patients, $48 \%$ ), a deterioration in clinical condition despite full intensive therapy (nine patients, $43 \%$ ) or failure to improve in association with CT evidence of pancreatic necrosis (two patients, $9 \%$ ). In contrast, Beger's group ${ }^{24}$ pursue a more aggressive policy. They report a series of 744 patients with acute pancreatitis. They instituted full supportive measures for system failure and operated if there was no evident clinical improvement within 3 days. Operation was also performed if there was a deterioration despite intensive care. Ninety-five patients were operated of whom 14 had fever and leucocytosis and persisting abdominal symptoms. Eighty-three per cent of patients had CT confirmation of necrotizing pancreatitis. In this series the median duration of conservative therapy was 5 days.

The operative mortality in these two series was very different. Eight of 21 (38\%) of the Glasgow patients died, compared with eight of 95 in the German series. However, the mortality related to the whole group of patients with acute pancreatitis was similar in the two series (less than $2 \%$ ). It therefore seems that surgery may be delayed beyond the first week in most patients, with no ill effect on overall mortality. This delay allows many patients to recover without surgery, and it allows time for the acute systemic effects of pancreatitis to subside. The surgeon must, however, beware of too long a delay, and any patient who continues to deteriorate on the intensive care unit should undergo surgical exploration.

The series from Atlanta ${ }^{23}$ emphasizes the virtue of conservative therapy unless there is a clear indication for surgery. Conservative management was successful in all but one patient with extensive necrosis without infection, although ultimately surgery was required in all those who had infected necrosis. The overall mortality in 194 patients was again $2 \%$. Table II summarizes the indications and appropriate timing of surgery for pancreatic necrosis.

At any time in the course of acute pancreatitis, and especially in the early stages, there may be evidence of peritonitis. This is uncommon, but it does require urgent assessment and surgery if appropriate. Colonic necrosis may occur in 1 or $2 \%$ of attacks of pancreatitis. This serious complication has a mortality greater than $50 \%{ }^{25}$ and constitutes a surgical emergency. At laparotomy, in addition to exposing the pancreas and draining or debriding the retroperitoneum as appropriate, allo necrotic colon must be excised and the ends exteriorized. This course of action is the most likely to salvage the patient's life.

\section{Pancreatic abscess}

This complication is probably a late consequence of infected peripancreatic necrosis. It is diagnosed by the combination of swinging pyrexia and ultrasonographic or CT evidence of a collection. Surgical drainage is indicated immediately the diagnosis has been made. ${ }^{26,27}$ Necrosectomy, debridement and

Table II Timing of surgery for pancreatic and peripancreatic necrosis

\begin{tabular}{|c|c|c|}
\hline Timing & Clinical situation & Indication for surgery \\
\hline $0-7$ days & $\begin{array}{l}\text { Severely ill patients with } \\
\text { full supportive therapy }\end{array}$ & Further deterioration \\
\hline $7-10$ days & As above & No improvement \\
\hline $7-10$ days & $\begin{array}{l}\text { Patient stable } \\
\text { CT shows extensive necrosis }\end{array}$ & $\begin{array}{l}\text { Infected necrosis } \\
\quad \text { (aspiration and culture) }\end{array}$ \\
\hline 10 days or more & General condition stable & $\begin{array}{l}\text { Persisting pyrexia or } \\
\quad \text { leucocytosis } \\
\text { Gastric outlet obstruction } \\
\text { Persisting pain }\end{array}$ \\
\hline Any time & Evidence of uncontrolled sepsis & Mandatory \\
\hline Any time & Clinical improvement & Not indicated \\
\hline
\end{tabular}


closed drainage give excellent results. ${ }^{27}$ Surgery is necessary to deal with these complex, often loculated abscesses, which frequently contain incompletely broken down necrotic material. Even those who are enthusiastic for percutaneous drainage of intra-abdominal abscess report poor results for pancreatic abscess. ${ }^{28}$

\section{Prophylaxis against infection}

After a decade of nihilism in the face of pancreatic necrosis, there is now some optimism that infection may be preventable in this condition. Earlier studies of antibiotic prophylaxis probably failed because the agents used did not penetrate adequately into pancreatic tissue. Several modern antimicrobial agents can penetrate well into pancreatic tissue..$^{29-31} \mathrm{~A}$ preliminary report indicates that imipenem may reduce the rate of infection in patients with necrosis. ${ }^{32}$ Encouraging results have also been demonstrated in a Dutch multicentre study of selective gut decontamination in patients with severe pancreatitis (Dr E.J.T. Luiten, personal communication). Further details of these studies are awaited with interest.

\section{Pancreatic pseudocyst}

The management of pancreatic pseudocyst has been confused by the increased availability of diagnostic imaging such as ultrasonography and CT. This has led to the detection of fluid collections around the pancreas at an early stage in the course of an attack of acute pancreatitis, and these may be erroneously diagnosed as pseudocysts. The vast majority of these collections will resolve spontaneously and it is therefore not surprising that early intervention such as percutaneous aspiration has a high success rate. ${ }^{33} \mathrm{~A}$ pseudocyst should be defined as a collection of fluid in the region of the pancreas enclosed by a fibrous tissue wall. This diagnosis implies a loculated chronic fluid collection, and the term pseudocyst is inappropriate during the first 2 weeks after the onset of symptoms.

A pseudocyst will be diagnosed when a patient has recovered from the acute phase of pancreatitis, but still complains of abdominal pain. Persisting elevation of serum amylase may suggest the diagnosis, which is confirmed initially by ultrasonography. This examination may be sufficient to define the relationships between the pseudocyst, and the pancreas and other structures. CT gives a very clear anatomical image, and may be required if intervention is contemplated.

The initial management of all pseudocysts is conservative. Spontaneous regression will occur in
$20-50 \%$ of patients during the first 6 weeks of observation. ${ }^{34-36}$ During this period only those cysts which are enlarging and severely symptomatic should be operated on. Recently it has become clear that there may be some benefit in further conservative management beyond 6 weeks $^{34}$ in contrast to the traditional teaching that resolution is rare beyond this time..$^{33}$ If the patient remains well and the cyst has not increased in size at 6 weeks, then a further period of 6 weeks observation is justified, irrespective of the size of the cyst. Cysts greater than $6 \mathrm{~cm}$ in size do have a higher risk of complications, but in the absence of symptoms this is insufficient to justify operation at 6 weeks in every patient. ${ }^{34}$

The assessment of patients for intervention should include pancreatography. However, endoscopic retrograde pancreatography (ERP) should be planned as an immediately preoperative investigation because of the risk of introducing infection into a cyst which communicates with the pancreatic duct. The interventional strategy can be determined from the appearances of the pancreatogram. ${ }^{37,38}$ If the pancreatic duct is normal and there is no communication with the cysts then percutaneous drainage will probably be successful, but if the pancreatic duct is abnormal, particularly if there is a communication with the cyst, then surgical management will be required. We have recently reviewed the management of pancreatic pseudocysts in detail, with particular reference to the choice between surgical and percutaneous techniques. ${ }^{39}$

\section{Conclusion}

The correct timing of intervention in acute pancreatitis requires careful clinical judgement. The management of common bile duct stones is now clear. Every patient with pancreatitis should have an ultrasound examination on the morning after admission to detect gallstones. Gallstones may be suspected by a suggestive clinical history or by elevated liver enzymes on admission, in addition to ultrasonography. All patients suspected of having gallstones should undergo ERC and possible sphincterotomy within 24-48 hours of admission. Patients with gallstones should undergo cholecystectomy before discharge from hospital, probably by the laparoscopic route.

The timing of intervention for pancreatic and peripancreatic necrosis and abscess is more difficult. It is our policy to delay intervention until 7-10 days after onset of symptoms and to operate at that time in those patients with documented necrosis who have continuing pain, gastric outlet obstruction, or fever and leucocytosis. Patients whose condition deteriorates at any stage despite 
full intensive care should undergo surgery. The decision to operate must be individualized and requires expertise and experience in the management of this condition. Similarly the management of pancreatic pseudocyst should be individualized and adapted to the clinical progress of the patient and findings on pancreatography. Patients with

\section{References}

1. Acosta, J.M. \& Ledesma, C.L. Gallstone migration as a cause of acute pancreatitis. $N$ Engl J Med 1974, 290: 484-488.

2. Acosta, J.M., Rossi, R., Galli, O.M.R., Argentina, R., Pellegrini, C.A. \& Skinner, D.B. Early surgery for acute gallstone pancreatitis: evaluation of a systematic approach. Surgery 1978, 83: 367-370.

3. Ranson, J.H.C. The timing of biliary surgery in acute pancreatitis. Ann Surg 1979, 189: 654-662.

4. Stone, H.H., Fabian, T.C. \& Dunlop, W.E. Biliary tract pathology in relation to time of operation. Ann Surg 1981, 194: 305-312.

5. Frey, C.F. A strategy for the surgical management of gallstones pancreatitis. In: Beger, H.G. \& Buchler, M. (eds) Acute Pancreatitis. Springer-Verlag, Berlin, 1987, pp. 242250.

6. Kelly, T.R. \& Elliott, D.W. Proper timing of surgery for gallstone pancreatitis. Am J Surg 1990, 159: 361-362.

7. Mackie, C.R., Wood, R.A.B., Preece, P.E. \& Cuschieri, A. Surgical pathology at early elective operation for suspected acute gallstone pancreatitis: preliminary report of a prospective clinical trial. Br J Surg 1985, 72: 179-181.

8. Rhodes, M., Armstrong, C.P., Longstaff, A. \& Cawthorn, S. Laparoscopic cholecystectomy with ERCP for acute gallstone pancreatitis. Br J Surg 1993, 80: 247.

9. Kim, U., Shen, H.'Y. \& Bodner, B. Timing of surgery for acute gallstone pancreatitis. Am J Surg 1988, 156: 393-396.

10. Kelly, T.R. \& Wagner, D. Gallstone pancreatitis: a prospective randomized trial of the timing of surgery. Surgery 1988 , 104: $600-605$

11. Safrany, L. \& Cotton, P.B. A preliminary report: urgent duodenoscopic sphincterotomy for acute gallstone pancreatitis. Surgery 1981, 89: 424-428.

12. Neoptolemos, J.P., Carr-Locke, D.L., London, N.J.M., Bailey, I.A., James, D. \& Fossard, D.P. Controlled trial of urgent endoscopic retrograde cholangiopancretography and endoscopic sphinterotomy versus conservative treatment for acute pancreatitis due to gallstones. Lancet 1988, ii: 979-983.

13. Nowak, A., Nowakowska-Dulawa, \& Rybicka, J. A prospective randomized trial of urgent endoscopic sphincterotomy vs conservative treatment for acute biliary pancreatitis (abp). Hepatogastroenterol 1990, 37 (Suppl II AS).

14. Fan, S.T., Lai, E.C.S., Mok, F., Lo, C.M., Zheng, S.S. \& Wang, J. Early treatment of acute biliary pancreatitis by endoscopic papillotomy. $N$ Engl J Med 1993, 328: 228-232.

15. Staritz, M. \& Meyer zum Buschenfelde, K.-H. Biliary pancreatitis: endoscopic diagnostic and therapeutic techniques. In: Beger, H.G. \& Buchler, M. (eds) Acute Pancreatitis. Springer Verlag, Berlin, 1987, pp. 233-241.

16. Block, S., Bittner, R. \& Beger, H.G. Sepsis indicators in acute pancreatitis. In: Beger, H.G. \& Buchler, M. Acute Pancreatitis. Springer Verlag, Berlin, 1987, pp. 164-170.

17. London, N.J.M., Neoptolemos, J.P., Lavelle, J., Bailey, I. \& James, D. Serial computed tomography scanning in acute pancreatitis: a prospective study. Gut 1989, 30: 397-403

18. Beger, H.G., Bittner, R., Block, S. \& Buchler, M. Bacterial contamination of pancreatic necrosis, a prospective clinical study. Gastroenterol 1986, 91: 433-438. complicated pancreatitis should be concentrated in the hands of interested specialists who can provide the appropriate combination of diagnostic and interventional radiology and surgical expertise to allow the most appropriate timing and the most appropriate intervention.

19. Banks, P.A. \& Gerzof, S.G. Indications and results of fine needle aspiration of pancreatic exudate. In: Beger, H.G. \& Buchler, M. (eds) Acute Pancreatitis. Springer Verlag, Berlin, 1987, pp. 171-174.

20. Block, S., Maier, W., Bittner, R., Buchler, M., Malfertheiner, P. \& Beger, H.G. Identification of pancreatic necrosis in severe acute pancreatitis: imaging procedures versus clinical staging. Gut 1986, 27: 1035-1042.

21. Reber, H.A. Surgical intervention in necrotizing pancreatitis. Gastroenterol 1986, 91: 479-482.

22. Bradley, E.L. \& Allen, K. A prospective longitudinal study of observation versus surgical intervention in the management of necrotizing pancreatitis. Am J Surg 1991, 161: 19-25.

23. Wilson, C., McArdle, C.S., Carter, D.C. \& Imrie, C.W. Surgical treatment of acute necrotizing pancreatitis. $\mathrm{Br} J$ Surg 1988, 75: 119-123.

24. Beger, H.G., Buchler, M., Bittner, R., Block, S., Nevalainen, T. \& Roscher, R. Necrosectomy and postoperative local lavage in necrotizing pancreatitis. Br $J$ Surg 1988, 75: 207-212.

25. Aldridge, M.C., Francis, N.D., Glazer, G. \& Dudley, H.A.F Colonic complications of severe acute pancreatitis. Br J Surg 1989, 76: 362-367.

26. Stricker, P.D. \& Hunt, D.R. Surgical aspects of pancreatic abscess. Br J Surg 1986, 73: 644-646.

27. Kune, G.A. \& Brough, W. Surgical intervention in severe acute pancreatitis: 476 cases in 20 years. Ann R Coll Surg Engl 1989, 71: 23-27.

28. Goletti, O., Lippolis, P.V., Chiarugi, M. et al. Percutaneous ultrasound guided drainage of abdominal abscesses. $\mathrm{Br} \mathrm{J}$ Surg 1993, 80: 336-339.

29. Buchler, M., Malfertheiner, P., Friess, H. et al. The penetration of antibiotics into human pancreas. Infection 1989, 17: 20-25.

30. Bassi, C., Lombardi, D., Bertazzoni, E. et al. Penetration of imipemen into pancreatic tissues during acute necrotizing form. Pancreas 1991, 6: 709.

31. Bradley, E.L. Antibiotics in acute pancreatitis. Am J Surg 1989, 158: 472-478.

32. Bassi, C., DiCarlo, V., Zerbi, A. et al. Role of imipenem in preventing infected necrosis during acute pancreatitis. Results of the Italian multicentre study. Digestion 1992, 52: 68.

33. Bradley, E.L., Clements, J.L. \& Gonzales, A.C. The natural history of pancreatic pseudocysts: a unified concept of management. Am J Surg 1979, 137: 135-141.

34. Imrie, C.W. \& Shearer, M.G. The diagnosis and management of pancreatic pseudocyst. In: Johnson, C.D. \& Imrie, C.W (eds) Pancreatic Disease, Progress and Prospects. Springer Verlag, London, 1991, pp. 299-309.

35. Agha, F.P. Spontaneous resolution of acute pancreatic pseudocysts. Surg Gynecol Obstet 1984, 158: 22-26.

36. Yeo, C.J., Bastidas, J.A., Lynch-Ngham, A., Fishman, E.K. Zimmer, M.J. \& Cameron, J.L. The natural history of pancreatic pseudocysts documented by computed tomography. Surg Gynecol Obstet 1990, 170: 411-417. 
37. Nordback, I., Auvinen, O., Aivo, O., Islauri, J. \& Teerenhovi, $O$. ERCP in evaluating the mode of therapy in pancreatic pseudocyst. HPB Surgery 1988, 1: 35-44.

38. D'Egidio, A. \& Schein, M. Pancreatic pseudocysts: a proposed classification and its management implications. $\mathrm{Br} J$ Surg 1991, 78: 981-984.
39. Moran, B., Rew, D. \& Johnson, C.D. Surgical debate: pancreatic pseudocysts should be treated by surgical drainage. Ann R Coll Surg Engl 1993, (in press). 